\title{
Psychometric evaluation of the Chinese version of the Toronto Hospital Alertness Test
}

\author{
Sha Li ${ }^{1}$, Daniel Yee Tak Fong ${ }^{1 *}$, Janet Yuen Ha Wong ${ }^{1}$, Kate Wilkinson ${ }^{2}$, Colin Shapiro², Edmond Pui Hang Choi ${ }^{1}$, \\ Bradley McPherson ${ }^{3}$, Cindy Lo Kuen Lam and Mary Sau Man Ip ${ }^{5}$
}

\begin{abstract}
Background: Alertness is an important part of attention which is different from the opposite of sleepiness. This study aimed to translate and assess the measurement properties of the Toronto Hospital Alertness Test (THAT) in Hong Kong Chinese population.

Methods: The standard forward-backward translation procedure and cognitive debriefing were conducted to obtain the Chinese THAT. One hundred Chinese adults completed the Chinese THAT, the Center for Epidemiological Studies Depression Scale (CES-D), the Pittsburgh Sleep Quality Index (PSQI), and the Athens Insomnia Scale (AIS) by telephone interviews.
\end{abstract}

Results: The factorial validity was assessed by confirmatory factor analysis, and the internal reliability was examined by coefficient omega. The two negatively worded items of the THAT had low factor loadings and were removed. One more item was removed based on the modification indices of the eight-item model. The remaining sevenitem THAT showed satisfactory unidimensionality with root mean square error of approximation (RMSEA) $=0.06$, standardized root mean square residual $(\mathrm{SRMR})=0.08$, and comparative fit index $(\mathrm{CFI})=1.00$. The coefficient omega of the seven-item Chinese THAT was 0.80 (95\% Cl: 0.74-0.86). Convergent validity was demonstrated with THAT moderately associated with CES-D $(r=-0.45, P<0.01)$, PSQI $(r=-0.40, P<0.01)$, and AIS $(r=-0.45, P<0.01)$.

Conclusions: The Chinese version of THAT demonstrated acceptable reliability and validity in a Chinese population. Keywords: Alertness, Confirmatory factor analysis, Reliability, Validity

\section{Background}

Attention, which in charge of information processing, is an important issue in education, psychology, and neuroscience studies [1]. Recently, researchers suggested investigating more specific components of attention, such as alertness, instead of the general process [1]. Alertness comprises of phasic alertness and tonic alertness [2]. Phasic alertness refers to the orienting response, and it changes relatively rapidly $[2,3]$. Tonic alertness is equivalent to vigilance, as well as to sustained attention, and it changes relatively slowly $[2$,

\footnotetext{
* Correspondence: dytfong@hku.hk

'School of Nursing, The University of Hong Kong, 21 Sassoon Road, Hong Kong, China

Full list of author information is available at the end of the article
}

3]. Alertness is commonly considered in medical sciences and is concerned with cognitive processing and is preferably assessed by physiological brain measurements [4], while it is described as the state of individual behavior with a focus on the interaction with the external or internal environment, such as sleep deprivation in behavioral sciences [5]. Many functionalists have resorted to define mental concepts functionally instead of philosophically or scientifically, which can make the concepts possible to be measured with external criteria [6]. Therefore, we refer to alertness as the responsivity to internal and external stimuli from the behavior approach in this article [6].

Low alertness levels may have an adverse impact on our daily life, but it has received limited attention in 
research. Impaired alertness is common not only in patients but also in the general population [7-9]. For example, truck drivers and health care workers are also at risk of impaired alertness [10-12]. It was estimated that 1.1 million crashes per year are associated with impaired alertness in the USA [12]. In addition to sleep deprivation, psychiatric disorders and prescribed or over-the-counter medications may also lead to impaired alertness [13, 14]. Impaired alertness may be associated with fatigue, low energy, drowsiness, reduced attention, and decreased concentration [9]. These symptoms and feelings can induce automobile accidents [15], psychological symptoms [16], physical symptoms [17], and decreased quality of life [9], as well as the higher likelihood of disability and increased risk of mortality [18]. In adolescents, daytime impaired alertness may induce depressed mood and impact academic performance, while depressed mood can reduce academic performance further [19].

A standardized measurement tool specific for alertness is needed to guide treatment and facilitate the development of psychopathology $[6,16]$. Two instruments have been developed for assessing alertness, namely the Toronto Hospital Alertness Test (THAT) and the ZOGIM Alertness (ZOGIM-A) Scale [6]. THAT aims to assess self-perceived alertness, including the ability to concentrate, to think of new ideas, and to focus on the task at hand over the past week, whereas ZOGIM-A aims to evaluate self-perceived impact or benefits of alertness and the extent of experiencing high alertness [6]. Most researchers are concerned with alertness level and the negative effects of impaired alertness, making the THAT more frequently used than the ZOGIM-A. The THAT in original English language demonstrated satisfactory reliability and validity. The Cronbach's coefficient alpha and test-retest reliability of THAT were 0.96 and 0.79 , respectively. Acceptable convergent validity was also shown, with a significant correlation with ZOGIM-A $(r=0.37, P<0.01)$ [6]. Although THAT is relatively new, it has already been used to evaluate the effect of interventions [20, 21] and also could distinguish selfreported anxious from non-anxious patients [16].

China is one of the most populous countries in the world. However, alertness has been less studied in Chinese populations, which may be partly attributed to the lack of a standardized measurement tool to assess alertness. Therefore, this study aimed to examine the psychometric performance of a Chinese language version of THAT in a Chinese population in Hong Kong.

\section{Methods}

\section{Linguistic validation of the Chinese THAT}

Figure 1 shows the linguistic validation procedure. We developed the Chinese version of THAT from the original English version using standard forward-backward procedures [22]. The original developer of the THAT, a local academic sleep specialist in charge of a sleep clinic, an academic statistician with prior experience in linguistic and psychometric evaluation of patient-reported outcomes, and local registered nurses who were also fluent in English were invited to comprise the expert committee. Then, two bilingual registered nurses who were native Chinese independently translated the THAT into traditional written Chinese. The academic statistician hosted a meeting with the two forward translators to agree on a consensus version. Any discrepancies on the two Chinese versions were discussed and resolved by the statistician with consensus of the two translators, and the preliminary consensus version was then reviewed by an academic sleep specialist in charge of a sleep clinic. Further modifications on the wordings were made. The final consensus version was then back-translated into English by another registered nurse who was not informed of the original English version. Any discrepancies between the backward and the original English versions were assessed by the expert committee, and revision of the Chinese version was made where needed.

\section{Participants}

One hundred subjects who were 18-year-old or above and were able to communicate in Chinese were targeted by telephone interview. Shift workers and those who were taking drugs for hypertension or sleep problems or who had mental illness were excluded. The THAT comprises 10 items. Using the standard estimate of 10 subjects per test item, 100 subjects would be adequate for assessing the construct validity of the Chinese version of THAT [23].

\section{Procedures}

Participants were recruited in a telephone survey by random digit dialing of household residential fixed numbers. Each randomly selected phone number was added or minus one or two to capture unlisted numbers. When there was more than one eligible subject in a household, the occupant with the next birthday was explained the study details and invited to provide oral consent. If the subject consented to participate in the study, the participant would be interviewed in Cantonese using a questionnaire written in traditional Chinese characters. Ethical approval for the project was obtained from the Institutional Review Board of the University of Hong Kong/Hospital Authority Hong Kong West Cluster (Ref no.: UW16-326).

\section{Measures}

Toronto hospital alertness test (THAT)

The 10-item THAT assessed self-perceived alertness over the past week [6]. Each item was graded on a $0-5$ Likert scale. After reverse coding the final two test items, a global 


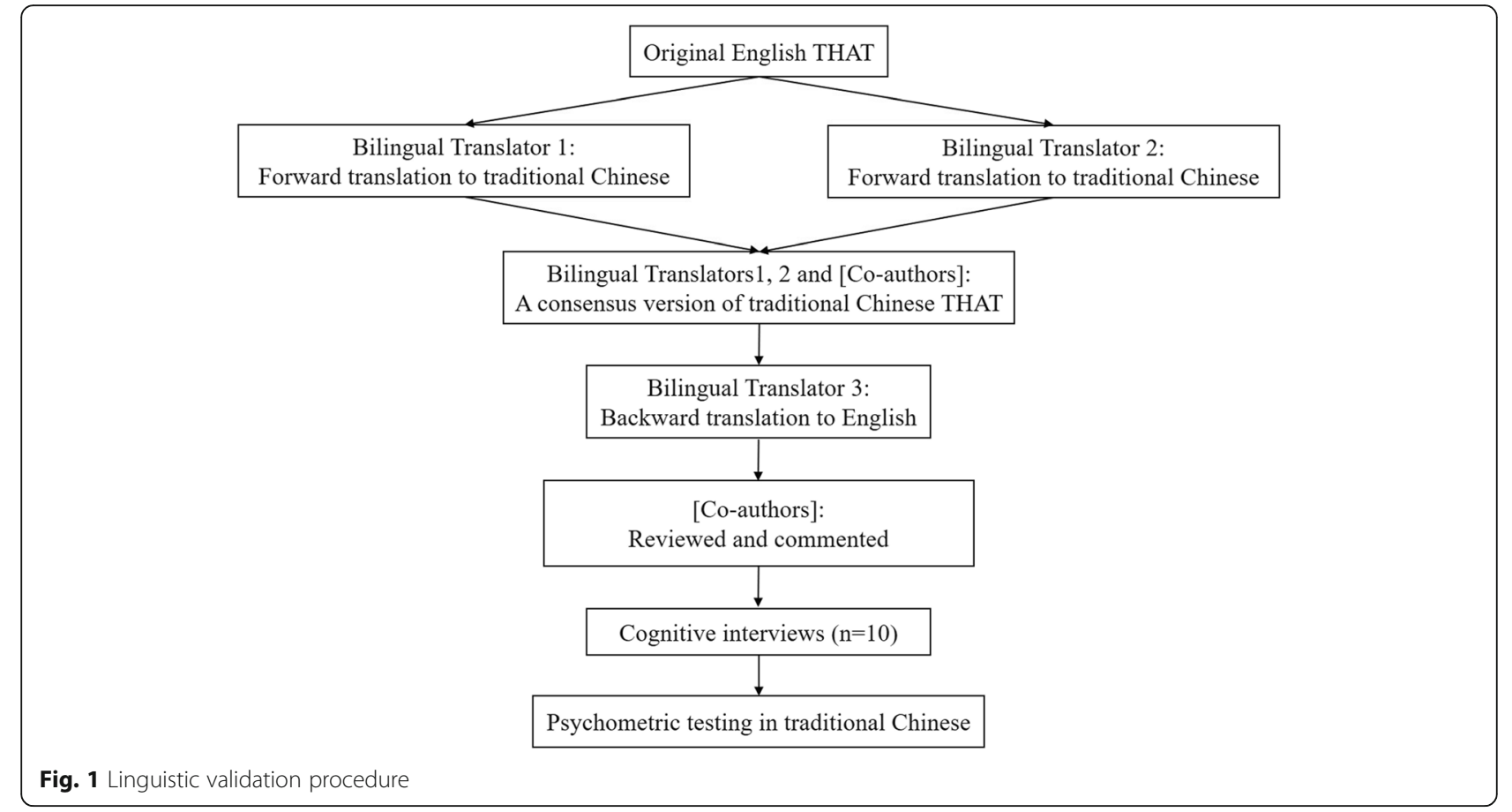

score was obtained as the response total. A higher global score corresponds to a higher level of alertness.

Center for Epidemiological Studies Depression Scale (CES-D) The self-rated CES-D scale comprises 20 items that evaluate the depressive symptoms [24]. It has been shown to be a valid tool to screen subjects with depression and to evaluate the severity of depressive disorders in a Hong Kong Chinese population [25]. The items were responded to using a $0-3$ Likert scale, according to the frequency of the symptoms. A higher score indicates more severe depression.

\section{Pittsburgh sleep quality index (PSQI)}

The 19-item PSQI questionnaire assesses sleep quality during the past month with diverse aspects relating to factors, including sleep latency and duration, subjective feeling of sleep quality and sleep efficiency, along with sleep-related problems [26]. The 19 items, each rated on a 0-3 scale, were grouped under seven components, namely: subjective sleep quality, sleep latency, sleep duration, habitual sleep efficiency, sleep disturbance, use of sleeping medications, and daytime dysfunction [26]. The component scores were the total scores of the corresponding items, which spans from 0 to 21 with a higher score indicating a worse sleep quality [27].

\section{Athens insomnia scale (AIS)}

The AIS is a self-report questionnaire for estimating sleep difficulty in the past month [28]. It contains eight items on sleep induction, awakenings during the night, final awakening earlier than desired, total sleep duration, the overall quality of sleep, as well as a sense of well-being and sleepiness during the day [29]. Participants graded their sleep quality from 0 to 3 according to the severity of their sleeping problems. The total score ranges from 0 to 24 , with a higher score corresponding to a worse sleep quality [29]. The internal consistency of AIS in this study was 0.84 .

\section{Statistical analysis}

The Chinese version of THAT was scored as the English version. The last two items of the original 10-item scale were reversed scored, and the floor and ceiling effects were then checked before analysis. If an overall value of greater than $15 \%$ for ceiling or floor effect exists, the validity, reliability, and responsiveness of a scale would be affected [30]. The factorial validity was examined through confirmatory factor analysis (CFA) by testing root mean square error of approximation (RMSEA) values, standardized root mean square residual (SRMR), and comparative fit index (CFI). The cut-off values were selected as 0.06 or below, 0.08 or below, and 0.95 or higher for RMSEA, SRMR, and CFI, respectively [31]. In the CFA models, the responses were taken as ordinal variables, and the diagonal weighted least squares (DWLS) estimator was used. DWLS is considered superior to robust maximum likelihood (MLR) when analyzing ordinal variables in latent variable modeling [32]. Furthermore, items with factor loadings smaller than 0.4 were suggested to be removed [33]. If the number of item changes, floor and ceiling effects are evaluated again before proceeding with analyses. Reliability of the Chinese version of THAT was assessed 
by omega and corrected item-scale correlations. The omega is considered the best alternative to Cronbach's $\alpha$ as the assumptions of Cronbach's $\alpha$, such as essentially tau-equivalence model, are usually violated [34]. The values of omega and corrected item-scale correlations were considered acceptable when greater than 0.7 [35] and 0.3 [23], respectively. When testing for convergent validity, for each subject with at most five $(50 \%)$ nonresponded items, the missing values were replaced with the average score of the remaining items. The Spearman rank correlation coefficients of the Chinese version of THAT with the CES-D, PSQI, and AIS were calculated to determine convergent validity. SPSS (version 23) and Rstudio-1.1.383 with the package "lavaan" [36] and "userfriendlyscience" [37] were adopted to perform data analysis. The significance level was set at 0.05 .

\section{Results}

Demographic characteristics and Chinese version of THAT scores

We interviewed 100 subjects. Their average age was 61 years old (Standard deviation: 17, range: 18-88), and 43 (43\%) were male. Twenty-five participants $(25 \%)$ had primary education or below, 44 (44\%) participants had secondary education, and 30 (30\%) participants had a bachelor's degree or above. There were $54(54 \%)$ retired participants, 17 (17\%) employees, 13 (13\%) homemakers, $6(6 \%)$ students, $4(4 \%)$ employers, 3 (3\%) self-employed participants, and 2 (2\%) job-seeking participants.

Table 1 shows the item characteristics. In total, 95 (95\%) participants completed all the items of THAT. Items 2,7 , and 8 had only one (1\%) missing value, while items 3 and 10 had 2 (2\%), and item 5 had 3 (3\%) missing values. Despite high floor or ceiling percentages in some items, the overall 10 -item scale score had only $1 \%$ ceiling and no floor effects.

\section{Factorial validity}

The 10-item one-factor model of THAT did not show satisfactory fit. The factors loadings of Item 9 "Extra effort was needed to maintain alertness" and Item 10 "In a boring situation, I would find my mind wandering" were 0.29 and 0.39 , respectively, both smaller than 0.4 . These two items were then removed, and the resulting eightitem one-factor CFA model was assessed.

The eight-item scale score had $4 \%$ ceiling and no floor effects. Modification index was highest in two pairs of error terms: (1) Item 3 "Fresh" and Item 4 "Energetic", (2) Item 3 "Fresh" and Item 5 "Able to think of new ideas". Incorporating the corresponding error covariances resulted in satisfactory fit. In view of the error covariances, a seven-item scale with Q3 removed was tested. Table 2 summarizes the fit indices of attempted CFA models. Figure 2 show the standardized coefficients of the seven-item one-factor CFA model.

\section{Scale reliability and convergent validity}

The coefficient omega of the seven-item scale was 0.80 (95\% CI: 0.74-0.86). The corrected item-scale correlations ranged from 0.30 to 0.68 .

Table 3 assesses the hypothesized association between THAT and the CES-D, PSQI, and AIS. The correlation was generally moderate in a range of 0.40 to 0.45 . All correlation coefficients were statistically significant.

\section{Discussion}

This study rigorously translated the THAT into traditional Chinese and evaluated its psychometric performance with a Cantonese speaking population. The unidimensional

Table 1 Summary of the Chinese version of THAT scores

\begin{tabular}{|c|c|c|c|c|c|c|c|}
\hline Items & Missing values (\%) & Mean (SD) & Min & Max & Median & Floor (\%) & Ceiling (\%) \\
\hline 1. Able to concentrate & 0 & $4.0(1.5)$ & 0 & 5 & 5 & 4 & 57 \\
\hline 2. Alert & 1 & $3.7(1.5)$ & 0 & 5 & 4 & 7 & 45 \\
\hline 3. Fresh & 2 & $2.3(2.0)$ & 0 & 5 & 2 & 30 & 21 \\
\hline 4. Energetic & 0 & $2.9(1.8)$ & 0 & 5 & 3 & 17 & 27 \\
\hline 5. Able to think of new ideas & 3 & $1.6(1.9)$ & 0 & 5 & 1 & 44 & 14 \\
\hline 6. Vision was clear noting all details (e.g., driving) & 0 & $3.9(1.6)$ & 0 & 5 & 5 & 8 & 57 \\
\hline 7. Able to focus on the task at hand & 1 & $4.2(1.2)$ & 0 & 5 & 5 & 2 & 58 \\
\hline 8. Mental facilities were operating at peak level & 1 & $3.8(1.4)$ & 0 & 5 & 4 & 3 & 46 \\
\hline 9. Extra effort was needed to maintain alertness & 0 & $3.4(2.0)$ & 0 & 5 & 5 & 19 & 52 \\
\hline 10. In a boring situation, I would find my mind wandering & 2 & $3.8(1.5)$ & 0 & 5 & 4 & 7 & 44 \\
\hline 10-item THAT & 5 & $33.4(9.7)$ & 3 & 50 & 36 & 0 & 1 \\
\hline 8-item THAT (items 9 and 10 deleted) & 5 & $26.3(8.6)$ & 3 & 40 & 28 & 0 & 4 \\
\hline 7-item THAT (items3, 9 and 10 deleted) & 4 & $24.1(7.4)$ & 3 & 35 & 26 & 0 & 5 \\
\hline
\end{tabular}


Table 2 Model fit indices in confirmatory factor analysis of the Chinese version of THAT

\begin{tabular}{|c|c|c|c|c|c|}
\hline Model & $x^{2}$ statistic & Degrees of freedom & RMSEA & SRMR & $\mathrm{CFI}$ \\
\hline 10-item one-factor & 76.13 & 35 & 0.11 & 0.11 & 0.97 \\
\hline 8-item one-factor & 40.86 & 20 & 0.11 & 0.10 & 0.98 \\
\hline 8-item one-factor (modified ${ }^{\mathrm{a}}$ ) & 22.61 & 18 & 0.05 & 0.07 & 1.00 \\
\hline 7-item one-factor & 18.54 & 14 & 0.06 & 0.08 & 1.00 \\
\hline
\end{tabular}

RMSEA root mean square error of approximation, SRMR standardized root mean square residual, CFI comparative fit index

${ }^{a}$ Incorporated error covariances: Q3 Q4 and Q3 Q5

Chinese version of THAT demonstrated satisfactory reliability and validity in Chinese people, in a Hong Kong setting.

The item non-responses were at most 3\%, demonstrating that the Chinese version of THAT was acceptable to the participants. However, the last two items, items 9 and 10, had low factor loadings that reflect their low association with the other items. This is consistent with a previous Canadian study [16]. This may be due to the questionnaire format, as the last two items are negatively worded, whereas the others are positively worded [16]. Individuals usually tend to agree more with positively worded items than negatively worded items [38]. Alternatively, Item 9 "Extra effort was needed to maintain alertness" may not be culturally consistent with a common practice of Chinese individuals, who may choose to rest rather than make an extra effort to maintain alertness $[39,40]$. For Item 10 "In a boring situation, I would find my mind wandering", studies had demonstrated that mind-wandering was not only an indicator of lacking alertness, but it can indicate future-oriented and creative thinking [41]. Furthermore, distinguishing different types or forms of mind-wandering may help explain these new findings [41].

Item 3 contributed to two error covariances that were required for satisfactory fit of the eight-item one-factor model. Firstly, the error covariance between Item 3 "Fresh" and Item 4 "Energetic" may be attributable to

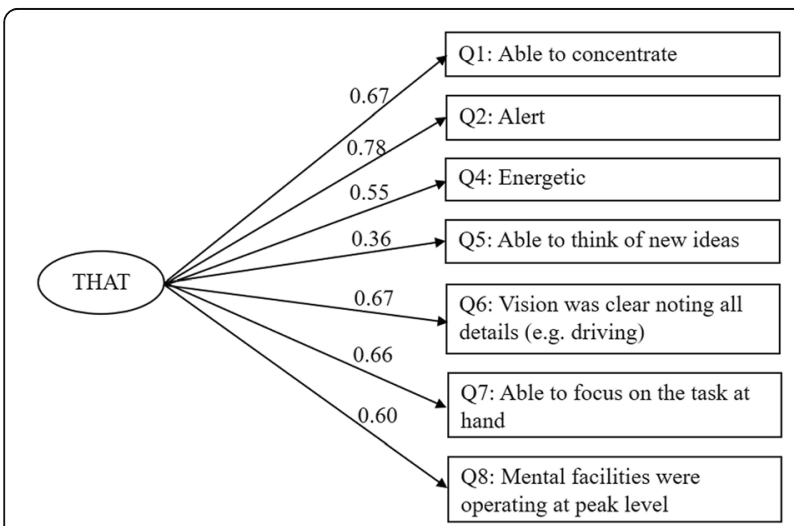

Fig. 2 Standardized coefficients of the seven-item Chinese version of Toronto Hospital Alertness Test (THAT) similar word meaning. In Chinese culture, a feeling of fresh or energetic is a common description of a good state of spirit and body. Secondly, the error covariance between Item 3 "Fresh" and Item 5 "Able to think of new ideas" may be due to the benefits from a good state of mind, such as positive affect and positive thinking [42]. In general, a state of "Fresh" partly comes from good sleep quality, which plays a critical role in regulating thinking ability, such as divergent thinking ability [43]. Therefore, the higher correlation due to similar meanings of Item 3 with Items 4 and 5 may make it unnecessary. After removing Item 3, the 7-item version fitted well. However, more research is valuable for evaluating Item 3.

The THAT in the Chinese language confirmed previously observed associations of alertness with CES-D, PSQI, and AIS scores, with correlation ranging from 0.40 to 0.45 . Depression has been shown to play an important role in regulating alertness [44]. Depressive individuals have lower levels of attention and vigilance, which can be attributed to decreased prefrontal cortex and anterior cingulate cortex (ACC) volume. These areas are partly responsible for processing visceral, effective, and attentional information [45]. Moreover, poor sleep quality also has a relationship with diminished alertness [19]. Poor sleep quality characterized by sleep fragmentation is correlated with sleepiness, and the effective treatment of fragmentation can help reduce sleepiness [46]. In terms of subjective sleep quality, restoration from sleep is well recognized [47], but nonrestorative sleep usually presents with higher sleepiness [48]. Lastly, insomnia is also responsible for impaired alertness [9]. Insomnia patients usually have poor sleep quality which can explain the relationship with alertness [49].

Despite our rigorous translation and psychometric assessment of the Chinese version of THAT, several

Table 3 Correlations between Chinese version of THAT score and other subjective scales

\begin{tabular}{llll}
\hline & CES-D & PSQI & AIS \\
\hline 7-item one-factor & $-0.45^{* *}$ & $-0.40^{* *}$ & $-0.45^{* *}$ \\
\hline
\end{tabular}

${ }^{* *} P<0.01$. THAT Toronto Hospital Alertness Test, CES-D Center for Epidemiological Studies Depression Scale, PSQI Pittsburgh Sleep Quality Index, AIS Athens Insomnia Scale 
limitations need to be mentioned. First, our sample was relatively small and larger sample size would be good to further explore the scale structure of the THAT and assess its measurement invariance across groups, such as sex. Second, it would also be desirable to examine testretest reliability. Lastly, the AIS has not been standardized validated in the Hong Kong Chinese adults. Nevertheless, the seven-item THAT in the Chinese language was found to be reliable and valid for assessing individual alertness in the present study.

\section{Conclusions}

The Chinese version of THAT is a reliable and valid instrument which can help future research in distinguishing and treating alertness without time-spending or resource-spending measurements.

\section{Abbreviations \\ AIS: Athens Insomnia Scale; CES-D: Center for Epidemiological Studies Depression Scale; CFA: Confirmatory factor analysis; CFI: Comparative fit index; DWLS: Diagonal weighted least squares; MLR: To robust maximum likelihood; PSQI: Pittsburgh Sleep Quality Index; RMSEA: Root mean square error of approximation; SD: Standard deviation; SRMR: Standardized root mean square residual; THAT: Toronto Hospital Alertness Test; ZOGIM- A: ZOGIM Alertness}

\section{Acknowledgements}

The authors gratefully thank Miss Tiffany Kwok and Mr. Cecil Wong, who conducted the forward and backward translations, respectively.

\section{Authors' contributions}

$S L$ did the data analysis and interpretation of data, drafted and revised the article and all other coauthors helped revise the article critically for important intellectual content. Furthermore, DF did the conception and design of the study, acquisition of data, analysis and interpretation of data. JW, KW, CS and $\mathrm{BM}$ also did the conception and design of the study. JW, EC, CL and Ml helped the acquisition of data.

\section{Funding}

This work was supported by a Seed Funding for Basic Research Grant from The University of Hong Kong (201511159061).

\section{Availability of data and materials}

No additional data are available.

\section{Ethics approval and consent to participate}

Ethical approval for the project was obtained from the Institutional Review Board of the University of Hong Kong/Hospital Authority Hong Kong West Cluster (Ref no.: UW16-326). Informed consent was obtained from all individual participants included in the study.

\section{Consent for publication}

Not applicable.

\section{Competing interests}

The authors declare that they have no conflict of interest.

\section{Author details}

${ }^{1}$ School of Nursing, The University of Hong Kong, 21 Sassoon Road, Hong Kong, China. ${ }^{2}$ Department of Psychiatry, University of Toronto, 399 Bathurst Street, Toronto, Canada. ${ }^{3}$ Human Communication, Development, and Information Sciences, The University of Hong Kong, Pokfulam Road, Hong Kong, China. ${ }^{4}$ Department of Family Medicine and Primary Care, The University of Hong Kong, 21 Sassoon Road, Hong Kong, China. ${ }^{5}$ Department of Medicine, The University of Hong Kong, 21 Sassoon Road, Hong Kong, China.
Received: 26 November 2019 Accepted: 15 April 2020

Published online: 05 May 2020

\section{References}

1. Burk, J. A., Blumenthal, S. A., \& Maness, E. B. (2018). Neuropharmacology of attention. Eur J Pharmacol, 835, 162-168.

2. Oken, B. S., Salinsky, M. C., \& Elsas, S. M. (2006). Vigilance, alertness, or sustained attention: Physiological basis and measurement. Clin Neurophysiol, 117(9), 1885-1901.

3. Nebes, R. D., \& Brady, C. B. (1993). Phasic and tonic alertness in Alzheimer's disease. Cortex, 29(1), 77-90.

4. Munde, V. S., et al. (2009). Alertness in individuals with profound intellectual and multiple disabilities: A literature review. Res Dev Disabil, 30(3), 462-480.

5. Thomas, M., et al. (2000). Neural basis of alertness and cognitive performance impairments during sleepiness. I. Effects of $24 \mathrm{~h}$ of sleep deprivation on waking human regional brain activity. J Sleep Res, 9(4), 335352.

6. Shapiro, C. M., et al. (2006). A new approach to the construct of alertness. J Psychosom Res, 60(6), 595-603.

7. Al-Abri, M. A., et al. (2018). Daytime sleepiness among young adult Omani car drivers. Sultan Qaboos Univ Med J, 18(2), e143-e148.

8. Videnovic, A. (2018). Disturbances of sleep and alertness in Parkinson's disease. Curr Neurol Neurosci Rep, 18(6), 29

9. Thorpy, M. J. (2005). Which clinical conditions are responsible for impaired alertness? Sleep Med, 6(Suppl 1), S13-S20.

10. Wilkinson, R., et al. (1989). Alertness of night nurses: Two shift systems compared. Ergonomics, 32(3), 281-292.

11. Smith-Coggins, R., et al. (2006). Improving alertness and performance in emergency department physicians and nurses: the use of planned naps. Ann Emerg Med, 48(5), 596-604 604 e591-593.

12. Rosekind, M. R. (2005). Underestimating the societal costs of impaired alertness: Safety, health and productivity risks. Sleep Med, 6(Suppl 1), S21S25.

13. Buffett-Jerrott, S. E., \& Stewart, S. H. (2002). Cognitive and sedative effects of benzodiazepine use. Curr Pharm Des, 8(1), 45-58.

14. Sforza, E., et al. (2002). Personality, anxiety and mood traits in patients with sleep-related breathing disorders: Effect of reduced daytime alertness. Sleep Med, 3(2), 139-145.

15. Aidman, E., et al. (2015). Real-time driver drowsiness feedback improves driver alertness and self-reported driving performance. Accid Anal Prev, 81, 8-13.

16. Shapiro, C., et al. (2017). Discriminating between anxious and non-anxious subjects using the Toronto hospital alertness test. Front Psychiatry, 8, 5.

17. Gustafsson, M. L., et al. (2018). Associations between daytime sleepiness, psychological symptoms, headache, and abdominal pain in schoolchildren. J Sch Nurs, 35, 279-286. https://doi.org/10.1177/1059840518774394.

18. Ng, W. L., Shaw, J. E., \& Peeters, A. (2018). The relationship between excessive daytime sleepiness, disability, and mortality, and implications for life expectancy. Sleep Med, 43, 83-89.

19. Short, M. A., et al. (2013). The impact of sleep on adolescent depressed mood, alertness and academic performance. J Adolesc, 36(6), 1025-1033.

20. Milewski-Lopez, A., et al. (2014). An evaluation of alertness training for older adults. Front Aging Neurosci, 6, 67.

21. Rogers, P. J., et al. (2013). Faster but not smarter: Effects of caffeine and caffeine withdrawal on alertness and performance. Psychopharmacology, 226(2) , 229-240.

22. Tsang, S., Royse, C. F., \& Terkawi, A. S. (2017). Guidelines for developing, translating, and validating a questionnaire in perioperative and pain medicine. Saudi J Anaesth, 11 (Suppl 1), S80-\$89.

23. Nunnally, J. C. (1978). Psychometric theory (2nd ed.). New York: McGraw-Hill.

24. Vilagut, G., et al. (2016). Screening for depression in the general population with the Center for Epidemiologic Studies Depression (CES-D): A systematic review with meta-analysis. PLoS One, 11(5), e0155431.

25. Chin, W. Y., et al. (2015). The psychometric properties of the Center for Epidemiologic Studies Depression Scale in Chinese primary care patients: Factor structure, construct validity, reliability, sensitivity and responsiveness. PLoS One, 10(8), e0135131.

26. Buysse, D. J., et al. (1989). The Pittsburgh sleep quality index: A new instrument for psychiatric practice and research. Psychiatry Res, 28(2), 193213. 
27. Chong, A. M. L., \& Cheung, C. K. (2012). Factor structure of a Cantoneseversion Pittsburgh sleep quality index. Sleep Biol Rhythms, 10(2), 118-125.

28. Kan, K. K. (2008). Validation of the insomnia severity index, Athens insomnia scale and sleep quality index in adolescent population in Hong Kong. The Universiy of Hong Kong Retrieved from http://hub.hku.hk/handle/10722/52015.

29. Soldatos, C. R., Dikeos, D. G., \& Paparrigopoulos, T. J. (2000). Athens insomnia scale: Validation of an instrument based on ICD-10 criteria. J Psychosom Res, 48(6), 555-560.

30. Terwee, C. B., et al. (2007). Quality criteria were proposed for measurement properties of health status questionnaires. J Clin Epidemiol, 60(1), 34-42.

31. Pallant, J. (2010). SPSS survival manual : A step by step guide to data analysis using SPSS (4th ed.). Maidenhead: Open University Press/McGraw-Hill.

32. Li, C. H. (2016). The performance of ML, DWLS, and ULS estimation with robust corrections in structural equation models with ordinal variables. Psychol Methods, 21(3), 369-387.

33. Kim, H., et al. (2016). Confirmatory and exploratory factor analysis for validating the phlegm pattern questionnaire for healthy subjects. Evid Based Complement Alternat Med, 2016, 2696019.

34. Dunn, T. J., Baguley, T., \& Brunsden, V. (2014). From alpha to omega: A practical solution to the pervasive problem of internal consistency estimation. Br J Psychol, 105(3), 399-412.

35. Gu, H. L., Wen, Z. L., \& Fan, X. T. (2017). Structural validity of the Machiavellian personality scale: A bifactor exploratory structural equation modeling approach. Personal Individ Differ, 105, 116-123.

36. Rosseel, Y., et al. (2012). Lavaan: Latent variable analysis. Retrieved from https://mirrors.tuna.tsinghua.edu.cn/CRAN/index.html.

37. Peters, G.-J., Verboon, P., \& Green, J. (2018). Userfriendlyscience: Quantitative analysis made accessible. Retrieved from https://mirrors.tuna.tsinghua.edu. cn/CRAN/index.html.

38. Fong, D. Y., et al. (2017). Measurement properties of the Chinese Weinstein noise sensitivity scale. Noise Health, 19(89), 193-199.

39. Baoying, H., et al. (2014). Association of napping and night-time sleep with impaired glucose regulation, insulin resistance and glycated haemoglobin in Chinese middle-aged adults with no diabetes: A cross-sectional study. BMJ Open, 4(7), e004419.

40. Chen, Y., et al. (2018). Characteristics and correlates of sleep duration, daytime napping, snoring and insomnia symptoms among 0.5 million Chinese men and women. Sleep Med, 44, 67-75.

41. Mooneyham, B. W., \& Schooler, J. W. (2013). The costs and benefits of mindwandering: A review. Can J Exp Psychol, 67(1), 11-18.

42. Fredrickson, B. L. (2001). The role of positive emotions in positive psychology. The broaden-and-build theory of positive emotions. Am Psychol, 56(3), 218-226.

43. Horne, J. A. (1988). Sleep loss and "divergent" thinking ability. Sleep, 11(6), 528-536.

44. Shahid, A., et al. (2016). The Toronto hospital alertness test scale: Relationship to daytime sleepiness, fatigue, and symptoms of depression and anxiety. Nat Sci Sleep, 8, 41-45.

45. Paelecke-Habermann, Y., Pohl, J., \& Leplow, B. (2005). Attention and executive functions in remitted major depression patients. J Affect Disord, 89(1-3), 125-135.

46. Roehrs, T., et al. (2016). Daytime sleepiness and alertness. In M. Kryger, T. Roth, \& W. Dement (Eds.), Principles and Practice of Sleep Medicine (6th ed. Amsterdam, pp. 39-48). Elsevier.

47. Harvey, A. G., et al. (2008). The subjective meaning of sleep quality: A comparison of individuals with and without insomnia. Sleep, 31(3), 383-393.

48. Neu, D., et al. (2010). Do 'sleepy' and 'tired' go together? Rasch analysis of the relationships between sleepiness, fatigue and nonrestorative sleep complaints in a nonclinical population sample. Neuroepidemiology, 35(1), 1-11.

49. Backhaus, J., et al. (2002). Test-retest reliability and validity of the Pittsburgh sleep quality index in primary insomnia. J Psychosom Res, 53(3), 737-740.

\section{Publisher's Note}

Springer Nature remains neutral with regard to jurisdictional claims in published maps and institutional affiliations.

\section{Submit your manuscript to a SpringerOpen ${ }^{\circ}$ journal and benefit from:}

- Convenient online submission

- Rigorous peer review

- Open access: articles freely available online

- High visibility within the field

- Retaining the copyright to your article

Submit your next manuscript at $\boldsymbol{\nabla}$ springeropen.com 